\title{
Editorial: The parallel squint
}

In the paper by Stein, Riddell, and Fowler in this issue new light is shed on the subject of dyslexia. It seems that some of the victims of this condition may be suffering from a motor rather than a sensory disorder. The disorder is identified as a failure of vergence to small targets on a synoptophore. Thus, when the small targets are viewed and, say, convergence is challenged, one eye is adducted but the other eye abducts a corresponding amount, so that the eyes remain parallel. This peculiar reaction seems to be something subtler than just a simple motor paresis of convergence, since performance is not so markedly affected when larger targets are used. We can assume therefore that convergence is possible but that, for reasons unknown, it fails with small targets, though visual acuity is normal.

One might be justified in saying that, since a convergent or divergent squint consists in a deviation from the position of the eyes which should be adopted in accordance with the object of regard, the present condition could be called a parallel squint.

We tend to take much for granted in our attitude to binocular vision and co-ordinated eye movements, and indeed our standard teaching on such subjects as the treatment of amblyopia by occlusion or on the surgical correction of strabismus tends to be somewhat simplistic. Because we have an impressive terminology relating to these problems we may feel, as clinicians, that we know more about them than is really the case. One has to admit, however, that our understanding of the subtleties of binocularity is still imperfect.

Perhaps we tend to forget that at the heart of the problem is the fact that the product of the images from the two eyes has to undergo a final synthesis into a single image at the level of consciousness. What the brain learns from the two eyes must accord with what is perceived via the other senses, and if there is dissonance between what appears to be and what ought to be something has to give way. This may be the explanation of, for example, why diplopia is more easily seen than confusion when the eyes are not both aimed at the object of regard. The brain can accept objects in pairs but not two dissimilar objects occupying the same space. An experiment with a vertical hand-held prism and two dropper bottles with different coloured labels placed one above the other on two shelves demonstrates this very well. An attempt to fuse one of the diplopic images of the red bottle with one of the green pair gives rise to the 'Cheshire Cat' phenomenon. Either the red label is seen or the green one but not both at the same time; as one becomes dominant the other fades out only to return again with a similar reversal of the colours. The time during which one colour remains dominant varies but is about $20-30$ seconds.

There are of course numerous other optical illusions which produce deceptive visual results for normal subjects. But what if one's eyes are not normal? It is obvious that in gross cases of squint optical illusions such as diplopia or misplacement can occur. But with subtler disorders of binocular control, such as that described in Stein, Riddell, and Fowler's article, one can readily believe that equally subtle optical illusions might occur, giving rise to difficulties with reading. One awaits further work on this subject with interest.

REDMOND J H SMITH 